\title{
Rates of climatic niche evolution are correlated with species richness in a large and ecologically diverse radiation of songbirds
}

Pascal 0. Title ${ }^{1,2 *}$ and Kevin J. Burns $^{1}$

\begin{abstract}
By employing a recently inferred phylogeny and museum occurrence records, we examine the relationship of ecological niche evolution to diversification in the largest family of songbirds, the tanagers (Thraupidae). We test whether differences in species numbers in the major clades of tanagers can be explained by differences in rate of climatic niche evolution. We develop a methodological pipeline to process and filter occurrence records. We find that, of the ecological variables examined, clade richness is higher in clades with higher climatic niche rate, and that this rate is also greater for clades that occupy a greater extent of climatic space. Additionally, we find that more speciose clades contain species with narrower niche breadths, suggesting that clades in which species are more successful at diversifying across climatic gradients have greater potential for speciation or are more buffered from the risk of extinction.
\end{abstract}

\section{Keywords}

Comparative methods, diversification, niche modelling, phenotypic rates, tanagers, thraupidae.

\section{INTRODUCTION}

To explain patterns of extant biodiversity, biologists have often sought to understand what causes certain groups of organisms to have more species than others. In many cases, speciose groups often have particular traits hypothesised to drive their diversification (Alfaro et al. 2009). Several factors may correlate with diversification rates, including morphology (Rabosky \& Adams 2012), geographic range size (Rabosky 2009), elevation (Kozak \& Wiens 2010b), latitude (Kozak \& Wiens 2007) and ecological opportunity (Mahler et al. 2010). High rates of climatic niche evolution have also been thought to drive diversification (Kozak \& Wiens 2007, 2010a). Generally, rate of diversification can be increased either by augmenting the rate of speciation and/or by reducing the rate of extinction. A possible mechanism that could lead to increased speciation is niche divergence, whereby incipient species adapt to different climates and subsequently become reproductively isolated (McCormack et al. 2010; Kozak \& Wiens 2010a). Decreased rates of extinction may result if some lineages have a greater ability to adapt quickly to new climatic niches, leading them to adapt rather than go extinct in a changing environment (Holt 2009; Kozak \& Wiens 2010a). Although a relationship between climatic niche evolution and species diversification has been hypothesised, this relationship has not been tested broadly, mainly due to a lack of a suitable methodology for quantifying environmental niches of multiple species in an evolutionary context.

Climatic niche modelling has been used extensively to quantify various aspects of species' ecological niches along specific environmental axes, and has increasingly been used in a phylogenetic context (Peterson et al. 1999; Eaton et al. 2008; Evans et al. 2009; Cooper et al. 2011). Physiological tolerances can be measured indirectly by quantifying species' occurrences along environmental axes (Peterson et al. 2011), which is often the only way such information can be recorded across large numbers of species. This approach assumes that a relationship exists between species' large-scale climatic distributions and their physiological tolerances (Holt 2009; Calosi et al. 2010). With a surge in availability of museum specimen records from natural history museums (Constable et al. 2010), and with the recent launch of observational databases (such as eBird [www.ebird.org]), large amounts of occurrence data can be compiled for species with relative ease. This data availability, coupled with the recent development of increasingly powerful data manipulation tools, has made large-scale studies of ecological niches feasible. However, few previous studies have investigated the relationship between ecological niche evolution and diversification rates (but see Kozak \& Wiens 2010b; Schnitzler et al. 2012).

In this study, we use climatic niche modelling in concert with a large phylogeny of songbirds known as tanagers (Thraupidae) to address hypotheses of ecological diversification. Tanagers are an ideal system in which to study the evolution of ecological niches. This clade of Neotropical birds consists of 371 species that have radiated across most of Central and South America (Fig. 1). They are found in 27 of the 29 terrestrial habitats identified in the Neotropics (Parker et al. 1996) and are distributed at elevations ranging from coastlines to Andean highlands. Representing $12 \%$ of all 


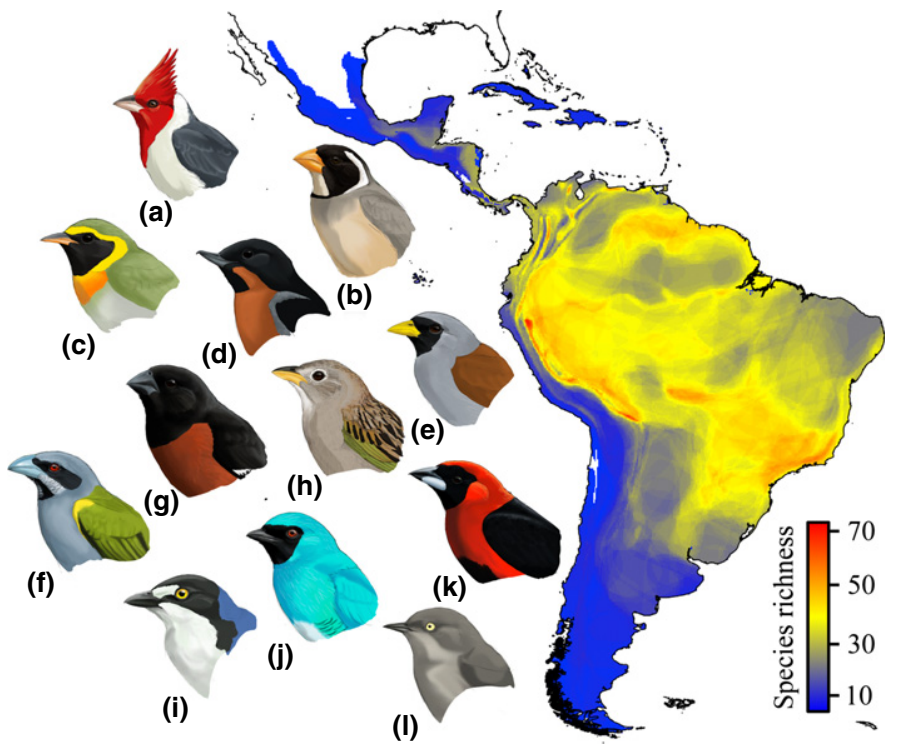

Figure 1 Overall tanager species richness mapped using species distribution range maps from Ridgely et al. (2007). Scale bar indicates the number of species. Representative species of all clades analysed in this study: (a) Paroaria coronata (Thraupinae), (b) Saltator aurantiirostris (Saltatorinae), (c) Hemithraupis guira (Hemithraupinae), (d) Diglossa brunneiventris (Diglossinae), (e) Incaspiza personata (Porphyrospizinae), (f) Parkerthraustes humeralis (Orchesticinae), (g) Sporophila angolensis (Sporophilinae), (h) Emberizoides herbicola (Emberizoidinae), (i) Nemosia pileata (Nemosiinae), (j) Tersina viridis (Dacninae), (k) Ramphocelus nigrogularis (Tachyphoninae) and (l) Hemispingus xanthophthalmus (Poospizinae). Illustrations courtesy of Mary Margaret Ferraro.

avian species in the region, tanagers are one of the most prominent groups in the Neotropics (Clements et al. 2013). Moreover, Thraupidae is the largest family of songbirds, representing nearly $10 \%$ of all songbird species. For decades, the phylogenetic limits of the group were uncertain (Sclater 1886; Storer 1969, 1970; Sibley \& Monroe 1990); however, recent molecular studies (Barker et al. 2013; Burns et al. 2014) have identified a monophyletic group that expands traditional ideas on species composition of the family. This new view of tanagers includes ecologically diverse species such as Darwin's Finches and Neotropical honeycreepers and includes species inhabiting a range of environments from dense tropical rainforest to high altitude $(>3000 \mathrm{~m})$ grasslands. Thus, the tanagers are an ideal group for an analysis of the relationship between diversification and climatic niche.

In this study, we test whether or not variation in diversification in tanagers can be explained by variation in rate of climatic niche evolution. That is, we test whether clades with more species also have higher rates of niche evolution. If shifts into new climatic regions have played a role in speciation in tanagers, then a relationship between rate of diversification and rate of ecological niche evolution might be expected (Kozak \& Wiens 2007; Sobel et al. 2010). We then discuss the relationship between diversification and niches in the context of geographic area and environmental space availability. If clades have higher rates of niche evolution, then they should have a greater propensity to occupy more environmental space. Therefore, we test for correlations between clade-specific niche evolutionary rate and clade-specific geographic range size, as range size is often considered to be a proxy for environmental space. In addition, as geographic range may not be perfectly representative of environmental space, we also test for correlations with niche volume. Because clades with greater species richness might be expected to occupy more environmental space as they diversify, we test for the correlation between clade richness and niche volume. We also examine the relationship between species niche breadth and clade diversification. Several different definitions exist for the niche, and some clades of tanagers have been studied in the context of various finer scale niche axes. Here, we define the niche as the set of broad climatic conditions that define the range of a species (Peterson et al. 2011).

\section{MATERIALS AND METHODS}

\section{Occurrence data acquisition}

Species-level sampling of niche data largely followed the taxonomy of Clements et al. (2013). In addition, we also modelled niches of Sicalis luteiventris and Poospiza whitii, two taxa treated as subspecies by Clements et al. (2013), but included in the phylogeny of Burns et al. (2014). Clements et al. (2013) treats these taxa as subspecies of Sicalis luteola and Poospiza nigrorufa respectively. For all 371 taxa we included, we downloaded the primary occurrence data, in the form of museum specimen records from four online data portals: ORNIS (http://www.ornisnet.org), GBIF (http:// www.gbif.org), IABIN (http://ara.inbio.ac.cr/) and speciesLink (http://splink.cria.org.br/). Queries through ORNIS also returned observational records from the eBird database (www.ebird.org). When specimen records were not completely available online, we requested data directly from museum curators. Our large dataset involved hundreds of thousands of species occurrence records and required the development of a new methodological pipeline (see online Supporting Information).

\section{Environmental data}

To train and project ecological niche models, we used the WorldClim dataset (Hijmans et al. 2005), which is a set of 19 bioclimatic variables representing various averages and extremes of temperature and precipitation, as interpolated from weather stations worldwide. The elevational dataset from WorldClim is derived from NASA's SRTM data (http:// www2.jpl.nasa.gov/srtm). Because of limitations from occurrence data, we used environmental grids at a 5 arc-min resolution (roughly $10 \times 10 \mathrm{~km}$ cells). In order to avoid possible confounding effects of correlated variables and over-fitting of niche models (Peterson et al. 2011), we calculated pairwise Pearson correlation coefficients between the environmental grids and excluded one of each pair of grids that were highly correlated. This correlation analysis was performed using the RASTER package in R (Hijmans \& van Etten 2012). We made sure that mean temperature and mean precipitation (bio 1 and bio 12 respectively) were kept, because of the ease of their interpretability, but forced isothermality and temperature 
seasonality to be dropped because of odd behaviour reported in ecological niche modelling applications (A. T. Peterson, pers. comm.). Seven bioclimatic variables and elevation were subsequently used in all modelling. These variables are annual mean temperature (bio 1), mean diurnal temperature range (bio 2), annual temperature range (bio 7), annual mean precipitation (bio 12), precipitation seasonality (bio 15), precipitation of the warmest quarter (bio 18), precipitation of the coldest quarter (bio 19) and elevation. Because the specimens used in this study were collected over the course of the last century, remote-sensing data could not be used because vegetation and land use has likely changed drastically in some areas, and no longer represents the conditions at the time of specimen collection.

\section{Climatic niche modelling}

We chose to construct niche models rather than directly use environmental conditions at the species localities. The suitability scores from the niche models can additionally be used to identify potentially erroneous species records. Niche modelling also avoids potentially detrimental effects of small sample sizes for species with few localities (for detailed niche modelling methods, see the online Supporting Information). We constructed climatic niche models with Maxent v3.3.3k (Phillips \& Dudík 2008). Maxent is a machine-learning method that uses an algorithm to find an optimal probability distribution given a set of environmental constraints. We chose to use Maxent among the available methods for niche modelling for a variety of reasons: absence data were lacking, Maxent has exhibited high performance relative to other methods (Elith et al. 2006; Wisz et al. 2008), it has been shown to produce robust results even with small sample sizes (Hernandez et al. 2006; Wisz et al. 2008), it has been extensively tested in the literature, and it can be implemented through $\mathrm{R}$ with the DISMO package (Hijmans et al. 2012).

We created an initial niche model using all occurrence points, and used the resulting model to identify the $5 \%$ of occurrence points with the lowest probability of occurrence. We then created a new niche model using $95 \%$ of the occurrence data. In this manner, we are more conservative with our interpretation of niche models, and we are also accounting for possible errors in the occurrence data used for model construction. We converted the probability distribution output by Maxent to a binary presence/absence raster using a least training presence threshold because the simple occurrence of the species is what is of interest here (Pearson et al. 2007; Waltari \& Guralnick 2009). Next, environmental data for all cells classified as present were extracted and the median was taken, so as to have representative values for each variable and for each species. This was executed with the RASTER package in $\mathrm{R}$ (Hijmans \& van Etten 2012).

\section{Phylogeny}

We used a posterior distribution of multi-locus, time-calibrated, species-level trees inferred using BEAST v1.7.1 (Burns et al. 2014). In these phylogenies, all but two species fell into one of 13 major clades, defined as the deepest strongly supported nodes in the phylogeny. These two species and the 13 major clades were each designated as one of 15 different subfamilies by Burns et al. (2014). Although poorly supported nodes with very short branches connect the subfamilies to each other, the phylogeny is characterised overall by strongly supported species relationships. Each of the subfamilies provides separate points in the phylogeny to compare niche evolutionary rate with diversification rate and other parameters. The phylogeny was also used to correct for phylogenetic relatedness between these subfamilies.

\section{Clade parameters}

To calculate rates of climatic niche evolution, we first took the median value for the eight environmental variables for each species, standardised the data to a mean of zero and standard deviation of one, and ran a principal components analysis (PCA) on the covariance matrix, in order to reduce dimensionality. To account for phylogenetic non-independence of the data in performing PCA, we employed a phylogenetically corrected PCA (Revell 2009), which uses the covariance structure of the phylogeny when transforming the data. PC axes 1 through 5 accounted for over $95 \%$ of the variation (Table 1) and so were kept for calculating rates of evolution. Next, the five PC axes were fit to two multivariate models of evolution, a Brownian Motion (BM) model and a global Ornstein-Uhlenbeck (OU) model, and the fits were compared with size-corrected $\mathrm{AIC}\left(\mathrm{AIC}_{\mathrm{c}}\right)$ in the $\mathrm{R}$ package OUCH (Butler \& King 2004). We chose to reduce our dataset via PCA because we experienced convergence difficulties with the model-fitting procedure with the original 8-variable dataset. As the OU model was preferred for several clades, we calculated rates from both models and found that the results were qualitatively similar, regardless of which model was used, so we present here the results from the multivariate BM model (see Supporting Information Tables A1 and A2 for results under an OU model). The trait evolution model was used to calculate the variance-covariance matrix for each clade. The diagonal elements of this matrix are the $\sigma^{2}$ values for each PC axis, which represent the phylogenetic rate of character evolution (O'Meara et al. 2006). The trace of the

Table 1 Variable loadings and per cent contribution for the phylogenetic principal components analysis used in the estimation of subfamily climatic niche rates of evolution

\begin{tabular}{lccccc}
\hline Variable & PC1 & PC2 & PC3 & PC4 & PC5 \\
\hline Elevation & 0.47 & 0.5 & 0.7 & -0.15 & -0.02 \\
Mean annual temp. & -0.52 & -0.74 & -0.22 & 0.23 & 0.11 \\
Mean temp. diurnal range & 0.9 & 0.06 & 0.15 & 0.34 & -0.09 \\
Temp. annual range & 0.81 & 0.15 & -0.41 & 0.37 & -0.1 \\
Annual precip. & -0.84 & -0.08 & 0.32 & 0.35 & -0.02 \\
Precip. seasonality & 0.58 & -0.67 & 0.42 & 0.08 & 0.12 \\
Precip. of warmest quarter & -0.48 & 0.47 & 0.13 & 0.47 & 0.54 \\
Precip. of coldest quarter & -0.76 & 0.01 & 0.27 & 0.34 & -0.48 \\
Eigenvalue & 0.24 & 0.09 & 0.07 & 0.05 & 0.03 \\
\% Variation & 49 & 18.43 & 13.9 & 9.44 & 6.43 \\
\hline
\end{tabular}

Temperature and precipitation are abbreviated as temp and precip respectively. 
matrix, which is the sum of these diagonal elements, gives a multivariate rate parameter (Kozak \& Wiens 2010a; Rabosky \& Adams 2012). This multivariate rate parameter was calculated across 1000 randomly selected trees from the post-burnin distribution from the BEAST analysis, and the median value was taken for each subfamily (Table 2).

We calculated clade-specific geographic area by overlaying the species distributions (Ridgely et al. 2007) from all species in each subfamily and combining these ranges as rasters, and then taking the area of those cells that indicated presence of the clade, using the R packages RASTER (Hijmans \& van Etten 2012) and GEOSPHERE (Hijmans \& Williams 2012) (Table 2). Because geographic space and environmental space are not equivalent, multivariate environmental breadth was also calculated for each clade. To do so, all unique environmental conditions were extracted for each clade. This was done by pooling the niche models from all species and extracting environmental conditions (the eight environmental variables selected earlier) for all presence cells. These cladespecific datasets were then standardised to a mean of zero and a variance of one. Principal components were calculated from the covariance matrix of these data, and the product of the eigenvalues (the standard deviations of the PC axes) was taken to represent the multivariate trait space occupied (Ricklefs \& Travis 1980). This PCA volume was calculated from 10,000 points. To robustly sample the niche volume for each clade, the median volume was taken from 1,000 resamplings of the multivariate environmental space. Following Fisher-Reid et al. (2012), we also calculated average proportional niche volume by calculating a similar species niche volume based on 1,000 points taken from each species' niche model, resampling 1,000 times, and dividing that by the clade's niche volume, and then averaging across all species of each clade. Thus, for each clade, we measured the average proportion of the clade's environmental niche that species occupy (Table 2).

\section{Correlation analyses}

To understand what factors are predictive of clade species richness, we examined correlations between richness, clade age, geographic area and niche volumes, using phylogenetic generalised least-squares regression (PGLS, Grafen 1989) (Table 3). As deriving the correlation structure from a BM model always yielded lower $\mathrm{AIC}_{\mathrm{c}}$ values than from an $\mathrm{OU}$ model $\left(\triangle \mathrm{AIC}_{\mathrm{c}}\right.$ range: $\left.0-13.32\right)$, we present results derived from a BM model (results using an OU correlation structure can be found in Table A3 in the Supporting Information). If diversification rates are changing through time, then looking for simple correlations with net diversification rate is inappropriate. Thus, before such correlations can be examined, the validity of constant-rate estimators of diversification must be verified (Rabosky \& Adams 2012). Rate constancy was tested with a diversification model-selection process described in Burns et al. (2014). As most clades in that analysis were fit best by a diversity-dependent model of diversification, we tested for correlations with species richness rather than with net diversification rate. Similarly, we tested for the appropriateness of using constant-rate models for niche rate by comparing the fits of a Brownian motion model, an OU model and an early burst model of trait evolution. To account for the multivariate nature of the data, we fit the models to each niche $\mathrm{PC}$ axis independently, and calculated multivariate $\mathrm{AIC}_{\mathrm{c}}$ by summing the likelihoods and incorporating an additional parameter for each PC axis.

Because clades with greater rate of niche evolution might be expected to occupy more geographic or environmental space, we checked for correlations between geographic area and niche rate, and a combination of geographic area and niche volume with niche rate. Finally, we looked for correlations between niche volume and richness to see if larger clades inhabit more environmental space. We log-transformed the data when necessary to obtain normality of the residuals. We accounted for phylogenetic uncertainty by running PGLS over a set of 1,000 randomly selected trees from the posterior distribution of the BEAST analysis. The median values of the output parameters are reported (Tables $3, \mathrm{~A} 1, \mathrm{~A} 2$ and A3). PGLS correlations were run with the NLME package in $\mathrm{R}$ (Pinheiro et al. 2012). A null expectation might be that geographic area increases with both greater species richness and greater niche volume, and we found that niche volume is highly correlated with geographic area (Pearson's correlation $\left.R^{2}=0.74, \quad P=0.001\right)$. Therefore, to avoid biases in our

Table 2 Diversification and climatic niche parameters for all major clades included in analyses

\begin{tabular}{|c|c|c|c|c|c|c|c|}
\hline Clade & Richness & Missing & Clade age & Niche rate & Clade area & Clade volume & Prop. niche volume \\
\hline Thraupinae & 102 & $4(13)$ & 0.817 & 9.282 & 17593144 & $8.76 \mathrm{e}-04$ & 0.059 \\
\hline Diglossinae & 64 & $0(11)$ & 0.874 & 38.696 & 14169494 & $5.19 \mathrm{e}-03$ & 0.010 \\
\hline Poospizinae & 44 & $1(6)$ & 0.771 & 16.627 & 10216155 & $1.67 \mathrm{e}-03$ & 0.031 \\
\hline Sporophilinae & 38 & $5(2)$ & 0.480 & 15.094 & 16580348 & $4.28 \mathrm{e}-04$ & 0.120 \\
\hline Tachyphoninae & 31 & $1(1)$ & 0.874 & 14.049 & 15975946 & $3.23 \mathrm{e}-04$ & 0.159 \\
\hline Saltatorinae & 16 & $0(4)$ & 0.78 & 5.810 & 15897059 & $5.20 \mathrm{e}-04$ & 0.099 \\
\hline Dacninae & 14 & $1(2)$ & 0.72 & 3.952 & 13726056 & $1.43 e-04$ & 0.359 \\
\hline Hemithraupinae & 9 & $0(1)$ & 0.786 & 8.752 & 12135063 & $1.55 \mathrm{e}-04$ & 0.332 \\
\hline Porphyrospizinae & 9 & $2(2)$ & 0.667 & 4.858 & 4142901 & $5.79 \mathrm{e}-04$ & 0.088 \\
\hline Emberizoidinae & 6 & $6(1)$ & 0.602 & 4.407 & 6664370 & $2.22 \mathrm{e}-04$ & 0.231 \\
\hline Nemosiinae & 5 & $1(1)$ & 0.629 & 7.347 & 12076374 & $2.84 \mathrm{e}-04$ & 0.181 \\
\hline Orchesticinae & 2 & $0(0)$ & 0.353 & 0.710 & 1976490 & $3.75 \mathrm{e}-06$ & 13.718 \\
\hline
\end{tabular}

Richness and clade age were estimated using all species sampled in the phylogeny, and niche parameters were estimated with species that had climatic niche data. The third column shows the number of species missing in the phylogeny, followed in parentheses by the number of species missing niche data. Clade area is in $\mathrm{km}^{2}$. 
Table 3 Results of phylogenetic least-squares regressions with a BM correlation structure, where niche rate is derived from a BM model of phenotypic evolution. Models within each block of the table were compared with the $\mathrm{AIC}_{\mathrm{c}}$ criterion and averaged with $\mathrm{Akaike}$ weights (wtAIC $\mathrm{C}_{\mathrm{c}}$ ). $\beta \pm \mathrm{SE}$ represents the regression coefficient and standard error from the PGLS models. The model found to be best has been bolded. Niche rate, clade richness, niche volume, and proportional niche volume were log-transformed. These correlations were tested across 1000 randomly sampled trees in order to account for phylogenetic uncertainty.

\begin{tabular}{|c|c|c|c|c|c|c|}
\hline Analysis & Effect & $\beta \pm \mathrm{SE}$ & $P$ & $\mathrm{AIC}_{\mathrm{c}}$ & $\Delta \mathrm{AIC}_{\mathrm{c}}$ & $\mathrm{wtAIC}_{\mathrm{c}}$ \\
\hline Richness $\sim$ clade age & Clade age & $4.81 \pm 1.76$ & 0.021 & 37.15 & 1.48 & 0.23 \\
\hline Richness niche rate & Niche rate & $\mathbf{0 . 9 7} \pm \mathbf{0 . 2 2}$ & 0.001 & 35.67 & $\mathbf{0}$ & 0.48 \\
\hline Richness $\sim$ clade area & Clade area & $<0.0001 \pm 0$ & 0.005 & 69.18 & 33.51 & 0 \\
\hline \multirow[t]{2}{*}{ Richness $\sim$ clade area + niche volume } & Clade area & $<0.0001 \pm 0$ & 0.039 & 69.46 & 33.79 & 0 \\
\hline & Niche volume & $0.37 \pm 0.13$ & 0.016 & & & \\
\hline \multirow[t]{2}{*}{ Richness $\sim$ niche rate + clade age } & Niche rate & $0.81 \pm 0.31$ & 0.027 & 36.72 & 1.05 & 0.29 \\
\hline & Clade age & $1.43 \pm 1.9$ & 0.47 & & & \\
\hline \multirow[t]{4}{*}{ Richness $\sim$ niche rate + clade age + clade area + niche volume } & Niche rate & $0.14 \pm 0.56$ & 0.81 & 80.82 & 45.15 & 0 \\
\hline & Clade age & $-0.08 \pm 2.02$ & 0.967 & & & \\
\hline & Clade area & $<0.0001 \pm 0$ & 0.139 & & & \\
\hline & Niche volume & $0.32 \pm 0.31$ & 0.336 & & & \\
\hline Niche rate $\sim$ clade area & Clade area & $<0.0001 \pm 0$ & 0.021 & 68.17 & 5.65 & 0.06 \\
\hline \multirow{2}{*}{ Niche rate clade area + niche volume } & Clade area & $<0.0001 \pm 0$ & 0.139 & 62.52 & $\mathbf{0}$ & 0.94 \\
\hline & Niche volume & $0.43 \pm 0.09$ & 0.001 & & & \\
\hline Prop. niche volume $\sim$ richness & Richness & $-1.18 \pm 0.29$ & 0.002 & & & \\
\hline
\end{tabular}

environmental space metric due to a geographic area effect, we examined correlations via multiple regression. Using this approach, the influence of environmental space on the dependent variable can be examined while the effect of area on environmental space is accounted for (Freckleton 2002).

\section{RESULTS}

We acquired over 340,000 occurrence records (after duplicates were removed) for tanagers from all databases that were accessed, of which $37 \%$ were observational records from eBird (www.ebird.org). The remaining records came from 135 different collections and institutions. After filtering steps were applied, a final dataset of 40,000 records remained, of which $50 \%$ were observational records. Island endemic species are problematic for niche modelling, because our understanding of which environments these species can and cannot tolerate is limited by the availability of environmental space on the island (Peterson et al. 2011). This holds especially for species that occupy the entirety of an island. Therefore, of the 371 species that comprise the tanagers, 29 species are island endemics and were removed from subsequent analyses. After further eliminating species that could not be modelled due to fewer than 6 occurrence records (40 species), and species that were not sampled in the phylogenetic inference (13 species), 285 species remained (Supporting Information Table A4). These species were used to study diversification rates across all tanagers. For the subfamily comparisons, three subfamilies were excluded: Catamblyrhynchinae and Charitospizinae because they are monotypic subfamilies, and Coerebinae because 26 of the 29 species in this clade are island endemics that could not be included in the analyses. Through phylogenetically corrected PCA, we were able to retain $97 \%$ of the variability in the data in the top five PC axes (Table 1). These axes do not lend themselves to any obvious interpretability, as most variables contribute to each axis.
As a whole, diversification across the phylogeny of Thraupidae is best fit by a diversity-dependent model (Burns et al. 2014). In addition, within Thraupidae, eight of the ten subfamilies examined are also best fit by diversity-dependent models (Burns et al. 2014). Clade age was significantly correlated with species richness (Table 3), although this correlation loses its statistical significance when the smallest clade, Orchesticinae, is removed (Table A1). These results imply that species accumulation in tanager subfamilies slows down over time. Therefore, constant-rate estimators are not appropriate for the study of tanager diversification, and species richness was used instead. In contrast, we did not recover evidence of variation in the rate of niche evolution within clades, as an early burst model of niche evolution fit best in only 1 out of 11 clades (Table A5).

We found a significant positive correlation between niche evolutionary rate and species richness (Table 3, Fig. 2a). The correlations involving rate of climatic niche evolution were qualitatively similar when environmental data directly extracted from localities were used, instead of values extracted via niche models (data not presented). We also found significant positive correlations between species richness and clade age, niche volume and clade geographic area. As some predictors of species richness are likely to be correlated, and since multiple variables might jointly explain species richness better than individual predictors, we also built multiple regression models, and compared models using $\mathrm{AIC}_{\mathrm{c}}$ and Akaike weights (Burnham \& Anderson 2002). We found that, although several variables are correlated with species richness, a model of species richness being predicted by variation in niche rate was strongly supported as the best model (Table 3). Other models, such as richness being correlated with clade age, niche volume or clade area, were found to be statistically significant. However, these models were ultimately less important relative to the strongly supported model when an $\mathrm{AIC}_{\mathrm{c}}$ model-selection approach was used. Clade age appears to be somewhat of an 

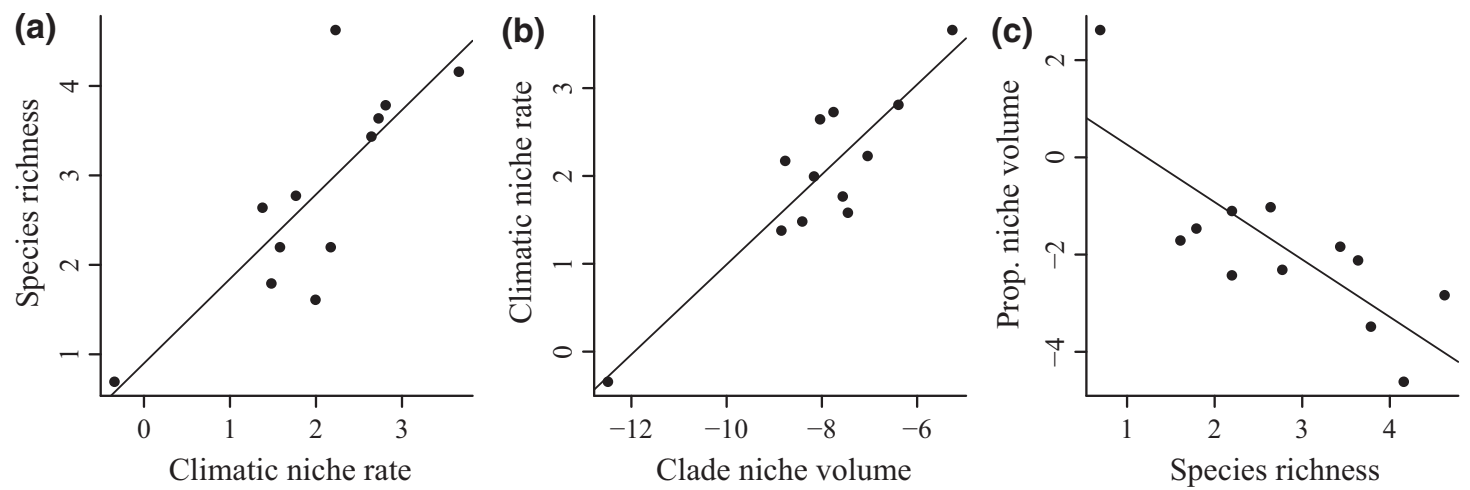

Figure 2 Relationship between (a) climatic niche rate and species richness, (b) clade niche volume and climatic niche rate and (c) average proportional niche volume and species richness. All variables were log-transformed.

important predictor, as the two other models that received support included clade age. Model-selection results were the same when different correlation structures were tested (Table A3) as well as when niche rate was derived from an OU model of trait evolution (Tables A1 and A2).

Clades that occupy larger geographic area were found to have higher rates of climatic niche evolution (Table 3 ). This might be expected because they would be encountering more environments and barriers than clades with more restricted ranges, thereby generating more opportunity for diversification (Kozak \& Wiens 2010b). However, a model that incorporates both area and environmental volume received much greater $\mathrm{AIC}_{\mathrm{c}}$ support. Additionally, we found that niche volume is highly correlated with niche rate (Fig. 2b), but geographic area is not, when the effect of geography on environmental space is controlled for in the multiple regression (Table 3).

Finally, proportional niche volume was negatively correlated with species richness (Fig. 2c), which shows that in larger clades, species have on average narrower niches relative to the niche space occupied by their clade.

\section{DISCUSSION}

We have shown that rate of climatic niche evolution is an important predictor of species richness, indicating that broadscale ecological parameters are important axes along which tanagers have diversified. Although incorporating clade age led to moderately supported models for predicting species richness, this appears to be at least somewhat driven by the inclusion of Orchesticinae, the smallest clade with 2 species, as this support weakens when this clade is left out of the analysis (Tables A1 and A2). Only a few previous studies have investigated the relationship between niche evolution and species richness. Our findings are in agreement with those of Kozak \& Wiens (2010a) on plethodontid salamanders. Though clade diversity was quantified differently, these authors also found support for rate of niche evolution being correlated with lineage diversification. Faced with significant climatic change, species from a clade with a low rate of niche evolution might be less likely to shift into new environmental space, and will run a greater risk of extinction (Wiens \& Graham 2005), leading to lower clade richness. The data presented here support this possibility: clades with lower rates of niche evolution are also those clades with the fewest species.

Clades with greater rate of climatic niche evolution are made up of more species, and these species occupy a greater geographic area and breadth of environmental space. As geographic area is accounted for in our measurement of niche volume via multiple regression, this result implies that larger clades occupy an amount of environmental space that is disproportionately larger than expected given the geographic area occupied by that clade. Ultimately, we would expect that rate of niche evolution should be best predicted by an axis that provides greater opportunity for diversification, which in this case is clade niche volume. As clades diversify, ecological opportunity will decline in the form of decreasing environmental space availability and lineage diversification will slow as a result (Mahler et al. 2010). Although we do not find evidence of a slowdown in niche rate to parallel the slowdowns in clade diversification, it is possible that niche space is still becoming saturated, but that this saturation is not leading to a slowdown in niche rate as defined by the early burst model of trait evolution. For example, niche space may be becoming saturated by species that are not each other's closest relatives.

The inverse correlation found between average proportional niche volume and species richness indicates that there is a link between a clade's inherent ability to carve out a subset of the available niche space (narrow niche breadth) and the clade's potential for speciation (Rundle \& Nosil 2005). Because this metric is calculated as a proportion of the clade's overall niche volume, clades with more species have on average subdivided the overall breadth of niche space occupied more finely. Such a correlation was not found for plethodontid salamanders (Fisher-Reid et al. 2012). Fisher-Reid et al. (2012) measured proportional niche breadth as the difference between minimum and maximum PCA-transformed bioclimatic variables, whereas we calculated the product of eigenvalues, also based on PCA-transformed bioclimatic variables, which is conceptually similar. To investigate whether or not this difference in results is methodological or ecological in nature, we calculated proportional niche breadth exactly as described in Fisher-Reid et al. (2012) and did not find a significant correlation for tanagers. Plethodontid salamanders have very different life histories as compared to tanagers and the nature of 
the association between these organisms and their climatic environment is likely very different. However, because a positive correlation between rate of climatic niche evolution and species diversity is found in both plethodontids and tanagers, climatic axes must be important for diversification in both cases.

Visual inspection of the graphs indicated that some clades depart from the overall trend (Fig. 2). For example, the tanager subfamily, Thraupinae, the most speciose tanager subfamily, has a substantially slower rate of niche evolution than would be expected, given its species richness (uppermost point, Fig. 2a). Thus, our results highlight groups where future research is warranted. Although we find correlations between diversification and niche metrics, other biological covariates may also play a role. It is possible that finer scale habitat and dietary niche characteristics for different clades lead to the broader climatic differences we have identified here. Additionally, other characteristics, such as evolutionary shifts in morphology or behaviour in tanagers might also influence diversification. Further research is warranted to gain an understanding of how different traits might influence diversification, alone and in concert.

This study is unique in the size of the clade being tested, and in the consideration of diversity-dependent models of diversification in the examination of climatic niches. Many studies of traits correlated with diversification have used net diversification rate, without considering whether or not such a metric is suitable (Rabosky \& Adams 2012). However, in many groups, including tanagers, diversity-dependent models best describe patterns of diversification (Burns et al. 2014); therefore, using net diversification rate is not appropriate. By using species richness in our analyses, we avoid this problem and properly examine the relationship between diversity and climatic niche evolution. Our results show that climatic niches play an important role in diversification across this speciose group of Neotropical birds. Some clades of tanagers are larger than others, and this is associated with shifts into new climatic niche space. This finding suggests that there is a link between speciation and the propensity of species to adapt to new climatic space. With speciose radiations, there is no a priori expectation that rates of niche evolution and species richness will follow such a pattern, as some other morphological or life history trait could be driving diversification, or diversification could result from non-adaptive, passive responses to geologic events. Our results indicate that niche evolution could play a fundamental role in our understanding of ecological controls on diversification, and should be investigated across a broad range of organisms. This paper should facilitate these studies by providing the necessary methodological pipeline to analyse niche evolution in large, geographically widespread clades.

\section{ACKNOWLEDGEMENTS}

We thank M. Faughender, R. House and S. Tuley for georeferencing assistance, A. T. Peterson for niche modelling training, and D. Rabosky for assistance with diversification analyses. We also thank C. Cicero and J. Wieczorek at UC Berkeley, and S. Cardiff at LSU for help in acquiring specimen records. We are indebted to Mary Margaret Ferraro for providing the tanager illustrations. We are grateful to all the scientific collectors, collections managers, curators, and associated faculty and staff at the numerous museums that provided the data used in this project. In particular, we are grateful to the $\mathrm{AMNH}$, the MCZ and the FMNH, which contributed significantly to our specimen dataset. For additional assistance, we thank L. Klicka, N. Mason, A. Shultz, A. Bohonak and P. Pryde. We are grateful to L. Klicka, N. Mason, A. Shultz, M. Grundler, several anonymous referees and the editor for insightful and constructive comments that improved this paper.

\section{FUNDING}

This research was funded in part by the National Science Foundation (IBN-0217817, DEB-0315416, and DEB1354006) and by the Mabel Myers Memorial Scholarship (SDSU).

\section{AUTHORSHIP}

P.O.T and K.J.B. designed the study; P.O.T. collected the data and conducted the analyses. P.O.T. and K.J.B wrote the paper.

\section{REFERENCES}

Alfaro, M.E., Santini, F., Brock, C., Alamillo, H., Dornburg, A., Rabosky, D.L., et al. (2009). Nine exceptional radiations plus high turnover explain species diversity in jawed vertebrates. PNAS, 106, 13410-13414.

Barker, F.K., Burns, K.J., Klicka, J., Lanyon, S.M. \& Lovette, I.J. (2013). Going to extremes: contrasting rates of diversification in a recent radiation of new world passerine birds. Syst. Biol., 62, 298-320.

Burnham, K.P. \& Anderson, D.R. (2002). Model Selection and Multimodel Inference. Springer, New York.

Burns, K.J., Shultz, A.J., Title, P.O., Mason, N.A., Barker, F.K., Klicka, J., et al. (2014). Phylogenetics and diversification of tanagers (Passeriformes: Thraupidae), the largest radiation of Neotropical songbirds. Mol. Phylogenet. Evol., 75, 41-77.

Butler, M. \& King, A. (2004). Phylogenetic comparative analysis: a modeling approach for adaptive evolution. Am. Nat., 164, 683-695.

Calosi, P., Bilton, D.T., Spicer, J.I., Votier, S.C. \& Atfield, A. (2010). What determines a species' geographical range? Thermal biology and latitudinal range size relationships in European diving beetles (Coleoptera: Dytiscidae). J. Anim. Ecol., 79, 194-204.

Clements, J.F., Schulenberg, T.S., Iliff, M.J., Sullivan, B.L., Wood, C.L. \& Roberson, D. (2013). The eBird/Clements checklist of birds of the world: Version 6.8. Downloaded from http://www.birds.cornell.edu/ clementschecklist/download/

Constable, H., Guralnick, R.P., Wieczorek, J., Spencer, C. \& Peterson, A.T. (2010). VertNet: A new model for biodiversity data sharing. PLoS Biol., 8, e1000309.

Cooper, N., Freckleton, R.P. \& Jetz, W. (2011). Phylogenetic conservatism of environmental niches in mammals. Proc. R. Soc. B, 278, 2384-2391.

Eaton, M., Soberón, J. \& Peterson, A.T. (2008). Phylogenetic perspective on ecological niche evolution in American blackbirds (Family Icteridae). Biol. J. Linn. Soc., 94, 869-878.

Elith, J., Graham, C.H., Anderson, R.P., Dudík, M., Ferrier, S., Guisan, A., et al. (2006). Novel methods improve prediction of species' distributions from occurrence data. Ecography, 29, 129-151.

Evans, M., Smith, S.A., Flynn, R. \& Donoghue, M.J. (2009). Climate, niche evolution, and diversification of the "bird-cage" evening 
primroses (Oenothera, sections Anogra and Kleinia). Am. Nat., 173, 225-240.

Fisher-Reid, M.C., Kozak, K.H. \& Wiens, J.J. (2012). How is the rate of climatic-niche evolution related to climatic-niche breadth? Evolution, 66, 3836-3851.

Freckleton, R.P. (2002). On the misuse of residuals in ecology: regression of residuals vs. multiple regression. J. Anim. Ecol., 71, 542-545.

Grafen, A. (1989). The phylogenetic regression. Philos. T. Roy. Soc. B, $326,119-157$.

Hernandez, P., Graham, C.H., Master, L. \& Albert, D. (2006). The effect of sample size and species characteristics on performance of different species distribution modeling methods. Ecography, 29, 773-785.

Hijmans, R.J. \& van Etten, J. (2012). raster: Geographic analysis and modeling with raster data. $\mathrm{R}$ package version 1.9-92.

Hijmans, R.J. \& Williams, E. (2012). geosphere: Spherical trigonometry. R package version $1.2-19$.

Hijmans, R.J., Cameron, S., Parra, J.L. \& Jones, P. (2005). Very high resolution interpolated climate surfaces for global land areas. Int. J. Climatol., 25, 1965-1978.

Hijmans, R.J., Phillips, S.J., Leathwick, J.R. \& Elith, J. (2012). dismo: Species distribution modeling. $\mathrm{R}$ package version 0.7-17.

Holt, R.D. (2009). Bringing the Hutchinsonian niche into the 21st century: ecological and evolutionary perspectives. PNAS, 106, 1965919665.

Kozak, K.H. \& Wiens, J.J. (2007). Climatic zonation drives latitudinal variation in speciation mechanisms. Proc. R. Soc. B, 274, 2995.

Kozak, K.H. \& Wiens, J.J. (2010a). Accelerated rates of climatic-niche evolution underlie rapid species diversification. Ecol. Lett., 13, 13781389.

Kozak, K.H. \& Wiens, J.J. (2010b). Niche conservatism drives elevational diversity patterns in Appalachian salamanders. Am. Nat., 176, 40-54.

Mahler, D.L., Revell, L.J., Glor, R.E. \& Losos, J.B. (2010). Ecological opportunity and the rate of morphological evolution in the diversification of Greater Antillean anoles. Evolution, 64, 2731-2745.

McCormack, J.E., Zellmer, A.J. \& Knowles, L.L. (2010). Does niche divergence accompany allopatric divergence in Aphelocoma jays as predicted under ecological speciation?: insights from tests with niche models. Evolution, 64, 1231-1244.

O'Meara, B.C., Ané, C., Sanderson, M. \& Wainwright, P.C. (2006). Testing for different rates of continuous trait evolution using likelihood. Evolution, 60, 922-933.

Parker, T.A., Stotz, D.F. \& Fitzpatrick, J.W. (1996). Ecological and distributional databases for Neotropical birds. In: Neotropical Birds: Ecology and Conservation. (eds Stotz, D.F., Fitzpatrick, J.W., Parker, T.A., Moskovits, D.K.). University of Chicago Press, Chicago, IL, pp. 113-436.

Pearson, R.G., Raxworthy, C., Nakamura, M. \& Peterson, A.T. (2007). Predicting species distributions from small numbers of occurrence records: a test case using cryptic geckos in Madagascar. J. Biogeog., 34, 102-117.

Peterson, A.T., Soberón, J. \& Sánchez-Cordero, V. (1999). Conservatism of ecological niches in evolutionary time. Science, 285, 1265-1267.

Peterson, A.T., Soberón, J., Pearson, R.G., Anderson, R.P., MartinezMeyer, E. \& Nakamura, M., et al. (2011). Ecological Niches and Geographic Distributions. Princeton University Press, Princeton, New Jersey, USA.

Phillips, S.J. \& Dudík, M. (2008). Modeling of species distributions with Maxent: new extensions and a comprehensive evaluation. Ecography, $31,161-175$
Pinheiro, J., Bates, D., DebRoy, S. \& Sarkar, D. \& R Development Core Team (2012). nlme: Linear and Nonlinear Mixed Effects Models. R package version 3.1-103.

Rabosky, D.L. (2009). Ecological limits on clade diversification in higher taxa. Am. Nat., 173, 662-674.

Rabosky, D.L. \& Adams, D. (2012). Rates of morphological evolution are correlated with species richness in salamanders. Evolution, 66, 18071818.

Revell, L.J. (2009). Size-correction and principal components for interspecific comparative studies. Evolution, 63, 3258-3268.

Ricklefs, R. \& Travis, J. (1980). A morphological approach to the study of avian community organization. Am. Nat., 97, 321-338.

Ridgely, R.S., Allnutt, T.F., Brooks, T., McNicol, D.K., Mehlman, D.W. \& Young, B.E., et al. (2007) Digital distribution maps of the birds of the western hemisphere, version 3.0. NatureServe, Arlington.

Rundle, H. \& Nosil, P. (2005). Ecological speciation. Ecol. Lett., 8, 336352.

Schnitzler, J., Graham, C.H., Dormann, C.F., Schiffers, K. \& Linder, H.L. (2012). Climatic niche evolution and species diversification in the Cape flora, South Africa. J. Biogeog., 39, 2201-2211.

Sclater, P.L. (1886). Catalogue of the Birds in the British Museum, Vol. XI. British Museum, London.

Sibley, C. \& Monroe, B. (1990). Distribution and Taxonomy of Birds of the World. Yale University Press, New Hauen, Connecticut.

Sobel, J.M., Chen, G.F., Watt, L.R. \& Schemske, D.W. (2010). The biology of speciation. Evolution, 64, 295-315.

Storer, R.W. (1969). What is a tanager? Living Bird, 8, 127-136.

Storer, R.W. (1970). Subfamily thraupidae. In: Check-list of Birds of the World. (ed Paynter, R.A. Jr). Museum of Comparative Zoology, Cambridge, MA, pp. 246-408.

Waltari, E. \& Guralnick, R.P. (2009). Ecological niche modelling of montane mammals in the Great Basin, North America: examining past and present connectivity of species across basins and ranges. $J$. Biogeog., 36, 148-161.

Wiens, J.J. \& Graham, C.H. (2005). Niche conservatism: integrating Evolution, Ecology, and Conservation Biology. Annu. Rev. Ecol. Evol. Syst., 36, 519-539.

Wisz, M.S., Hijmans, R.J., Li, J., Peterson, A.T., Graham, C.H., Guisan, A., et al. (2008). Effects of sample size on the performance of species distribution models. Divers. Distrib., 14, 763-773.

\section{SUPPORTING INFORMATION}

Additional Supporting Information may be downloaded via the online version of this article at Wiley Online Library (www.ecologyletters.com).

\section{Editor, Arne Mooers}

Manuscript received 7 August 2014

First decision made 10 September 2014

Second decision made 25 October 2014

Third decision made 23 December 2014

Manuscript accepted 22 January 2015 